\title{
Influence of Longitudinal Inertia of Mass Element on Non-Linear Component of Beam Natural Frequency
}

\author{
S. UZny, Ł. Kutrowski* And M. Osadnik \\ Czestochowa University of Technology, 42-201 Czestochowa, Poland \\ Doi: 10.12693/APhysPolA.138.302 \\ *e-mail: kutrowski@imipkm.pcz.pl
}

\begin{abstract}
In this paper the problem of non-linear vibrations of the beam was considered. A system with a mass element mounted at the end of the structure with a mass much larger than the mass of the beam was under consideration. This element during transverse vibrations generates additional longitudinal force associated with its inertia. The occurrence of this force results in the appearance of a nonlinear relationship between the amplitude of vibration of the system and its natural frequency. The boundary problem of non-linear vibrations was formulated on the basis of the Hamilton principle, taking into account the Bernoulli-Euler theory and the non-linear relationship between deformation and displacement (von Karman theory). Numerical simulations were carried out taking into account the impact of longitudinal inertia of the mass element on the non-linear component of natural frequency at different amplitudes of beam vibrations.
\end{abstract}

topics: free non-linear vibrations, beam, mass element

\section{Introduction}

In the case of mechanical structures exposed to periodically changing forces, an important element in the design process is the analysis of natural vibrations. Knowledge of the natural frequency allows one to make the necessary modifications to avoid adverse resonance phenomena. The increase in vibration amplitude (in the case of resonance) would cause a significant increase in wear and even structural damage.

Columns and beams are the basic mechanical systems for which the natural vibration analysis is carried out. In the case of columns, the problem of natural vibrations is related to the stability of the system. The columns subjected to the classic Euler load (load does not change the direction of operation during deflection) are divergent systems. In those kinds of systems, the zero value of the first natural frequency corresponds to the critical force. In literature one can find many publications corresponding to the subject of column and beam vibrations [1-11]. The considerations take into account a number of properties of these systems, including geometric parameters, type of mountings and method of loading. Research is also carried out using various theories of problem description (e.g., Euler, Timoshenko) or methods of their solutions. The analysis of the problem of free vibrations and stability of double tapered columns with elastic foundation and tip mass has been made in [2] reflecting the theory of Bernoulli-Euler and Timoshenko. The impact of loading head parameters on vibrations and column stability with damage by taking into account the specific load is considered in [3]. The problem of free vibration of the column by taking into account the Euler compression load and the impact of the local heat source is presented in [4]. In turn, free and forced vibrations of Timoshenko beams are analysed in [5], described by single difference equation at different boundary conditions. Publication [6] covers the matter of transverse vibrations of a beam with mass at free end. The studied system uses additional support at various distances from the rigid mounting. Free vibrations of beams with an intermediate sliding connection joint connected with a mass-spring system is also considered [7]. There, two types of additional systems are adopted: a harmonic oscillator with one and two degrees of freedom. The case of free and forced vibration of geometrically linear and non-linear beams when taking into account the occurrence of multi-cracks, is considered in [8]. Further, the analysis of forced vibrations of composite beams has been performed in [9] using the variable separation method. In fact, number of numerical calculations are carried out for various slenderness ratio and system mountings to validate the proposed method. The results are compared with exact elasticity solution and the FEM method. Publication [10] covers the analysis of free vibration problems perfectly clampedfree beams with additional stepwise eccentric distributed masses. Studies of free vibrations of multistepped beams are made [11] with the Adomian decomposition method (ADM). It is shown that ADM offers an accurate and effective method of free vibration analysis of multiple-stepped beams with arbitrary boundary conditions. 
We analyse the problem of free vibrations of double hinged beam system connected to the mass element $M$. This kind of system can represent a piston rod in a hydraulic cylinder, used in executive devices with a great mass. Hydraulic cylinder operates in a horizontal plane and its end is connected to latch. In this case, the longitudinal inertia of the mass $M$ has a significant impact on nonlinear vibrations of the beam. Such a formulation of the problem was not analysed in other works. In the problem formulated in this way, inertia generates additional forces in the case of vibrations, which introduce into the system nonlinearities depending on the vibration amplitude. Numerical simulations were carried out taking into account the influence of mass element inertia on the free vibrations of the system. Calculations were carried out for different levels of amplitudes.

\section{Boundary problem}

The system presented in Fig. 1 was considered. It is an articulated beam of length $l$, at the end of which is an element with mass $M$. The boundary problem was presented using dimensionless quantities:

$$
\begin{aligned}
& \xi=\frac{x}{l}, \quad \tau=\omega t, \quad \theta=\frac{A l^{2}}{J}, \\
& w(\xi, \tau)=\frac{W(x, t)}{l}, \quad u(\xi, \tau)=\frac{U(x, t)}{l}, \\
& k^{2}(\tau)=\frac{S(t) l^{2}}{E J}, \quad \Omega^{2}=\frac{\omega^{2}(\rho A) l^{4}}{E J},
\end{aligned}
$$

where $W(x, t)$ - displacement of the system in transverse direction, $U(x, t)$ - displacement of the system in longitudinal direction, $S(t)$ - internal force, $\omega$ - linear free vibrations frequency, $\rho$ - density of the beam, $A$ - cross-section area of the beam, $E J$ - flexural rigidity.

The formulation of the issue of natural vibrations was carried out using the Hamilton principle. After carrying out the necessary mathematical operations, the equations of motion of the system in the transverse (2) and longitudinal (3) directions are obtained:

$$
\begin{aligned}
& \frac{\partial^{4} w(\xi, \tau)}{\partial \xi^{4}}+\Omega^{2} \frac{\partial^{2} w(\xi, \tau)}{\partial \tau^{2}}=0 \\
& \frac{\partial}{\partial \xi}\left[\frac{\partial u(\xi, \tau)}{\partial \xi}+\frac{1}{2}\left(\frac{\partial w(\xi, \tau)}{\partial \xi}\right)^{2}\right]=0 .
\end{aligned}
$$

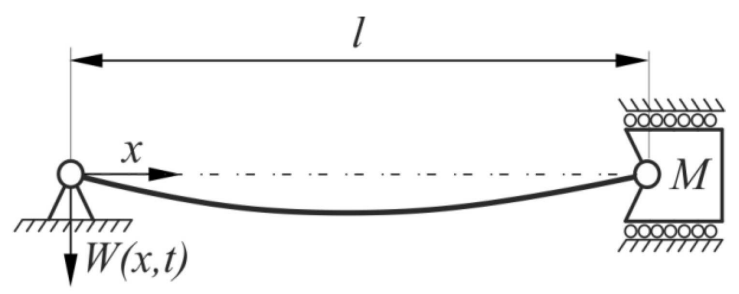

Fig. 1. Beam with the mass element.
After double integration, (3) gives:

$$
u(\xi, \tau)=\frac{-k^{2}(\tau) \xi}{\theta}-\frac{1}{2} \int_{0}^{1}\left(\frac{\partial w(\xi, \tau)}{\partial \xi}\right)^{2} \mathrm{~d} \xi
$$

The boundary conditions of the considered system are as follows:

$$
\begin{aligned}
& w(0, \tau)=0, \quad w(1, \tau)=0 \\
& \left.\frac{\partial^{2} w(\xi, \tau)}{\partial \xi^{2}}\right|_{\xi=0}=0,\left.\quad \frac{\partial^{2} w(\xi, \tau)}{\partial \xi^{2}}\right|_{\xi=1}=0, \\
& \frac{k^{2}(\tau) E J}{l^{2}}-\left.M \omega^{2} l \frac{\partial^{2} u(\xi, \tau)}{\partial \tau^{2}}\right|_{\xi=1}=0 .
\end{aligned}
$$

Due to non-linearity, the small parameter method is used to finally formulate the vibration problem. It consists in developing all nonlinear terms of the obtained equations into the power series of the small parameter of vibration amplitude $\varepsilon$. These series are as follows:

$$
\begin{aligned}
& w(\xi, \tau)=\varepsilon w_{1}(\xi, \tau)+\varepsilon^{3} w_{3}(\xi, \tau)+\ldots \\
& u(\xi, \tau)=+\varepsilon^{2} u_{2}(\xi, \tau)+\ldots \\
& k^{2}(\tau)=\varepsilon^{2} k_{2}^{2}(\tau)+\ldots \\
& \Omega^{2}=\Omega_{0}^{2}+\varepsilon^{2} \Omega_{2}^{2}+\ldots \\
& \omega^{2}=\omega_{0}^{2}+\varepsilon^{2} \omega_{2}^{2}+\ldots
\end{aligned}
$$

where:

$$
\begin{aligned}
& w_{1}(\xi, \tau)=\stackrel{(1)}{w}_{1}(\xi) \cos (\tau), \\
& w_{3}(\xi, \tau)=\stackrel{(1)}{w}_{3}(\xi) \cos (\tau)+\stackrel{(3)}{w}_{3}(\xi) \cos (3 \tau), \\
& u_{2}(\xi, \tau)=\stackrel{(2)}{u}_{2}(\xi) \cos (2 \tau), \\
& k_{2}^{2}(\tau)=\stackrel{(2)}{k}_{2}^{2} \cos (2 \tau) .
\end{aligned}
$$

Power series (6) (considering (7)) are substituted for differential equations and boundary conditions. After grouping the appropriate quantities relative to the powers of the small parameter, we obtain sequences of differential equations and the corresponding boundary conditions. On their basis, the following parameters can be determined:

- linear component of the natural frequency $\omega$,

- non-linear component of internal force $\stackrel{(2)}{k_{2}^{2}}$,

- non-linear component of natural vibrations frequency $\omega_{2}^{2}$.

\section{Results of numerical simulations}

The results of numerical calculations were presented using dimensionless parameters:

$$
\Omega^{*}=\sqrt{\Omega_{0}^{2}+\varepsilon^{2} \Omega_{2}^{2}}, \quad \zeta_{A}=\frac{\mathrm{amp}}{r},
$$

where $\Omega^{*}$ - parameter of the first natural vibration frequency, taking into account linear $\Omega_{0}$ and non-linear $\Omega_{2}$ parameters of the natural frequency, 

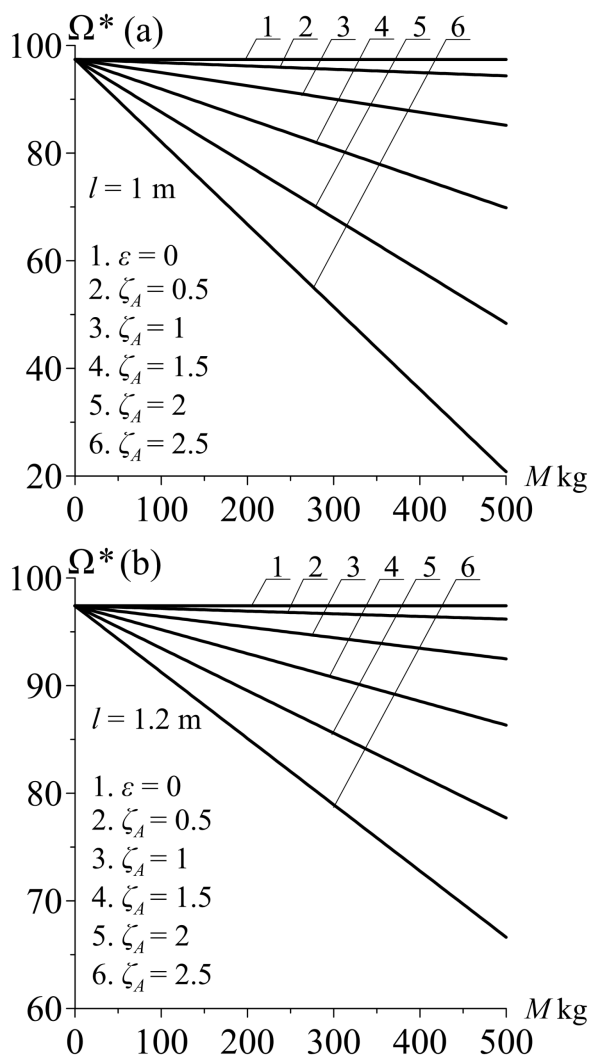

Fig. 2. Relation between natural frequency parameter and mass of the element for (a) $l=1 \mathrm{~m}$ and (b) $l=1.2 \mathrm{~m}$.

$\zeta_{A}$ — vibration amplitude parameter, amp - amplitude of system vibrations, $r$ - radius of gyration.

The calculations were carried out for two different lengths of the beam $l=1 \mathrm{~m}$ and $l=1.2 \mathrm{~m}$. Figure 2 presents the relationships between the natural vibration frequency parameter $\Omega^{*}$ and the mass of the additional element. Horizontal curves number 1 correspond to the zero value of the small parameter $\varepsilon$. It represents only the liner component of the free vibrations frequency parameter. The curves marked as $\langle 2 \ldots 6\rangle$ refer to subsequent values of the system vibration amplitude.

Based on the obtained results, it can be stated that as the mass of the element associated with the sliding support increases, the value of the system's own frequency parameter decreases. The dependencies $\Omega^{*}(M)$ shown on the graph are linear. It has also been observed that the frequency decreases as the vibration amplitude increases. Therefore, by increasing the amplitude of vibrations, the longitudinal inertia of the mass element occurring at the sliding end of the beam reduces the frequency of the system's own vibrations. The level of influence of parameters on the vibration frequency is much greater in the case of systems, characterised by smaller value of the system length. Comparing the relations in Fig. $2 \mathrm{a}$ and $2 \mathrm{~b}$ it can be stated that a small increase in the beam length (from $l=1 \mathrm{~m}$ to $l=1.2 \mathrm{~m}$ ) causes a great decrease in the value of the vibration frequency parameter.
Considering the vibrations with the highest amplitude value $\left(\zeta_{A}=2.5\right)$, with a mass $M=500 \mathrm{~kg}$, the reduction in system length by $0.2 \mathrm{~m}$ resulted in a decrease in the natural frequency from around $\Omega^{*}=65$ to $\Omega^{*}=20$.

\section{Conclusions}

In this paper, the problem of nonlinear beam vibrations has been formulated, taking into account the inertia of the mass element located at one of the ends of the system (at the end which is connected with the sliding support). It has been stated that the vibration amplitude and longitudinal inertia of the mass $M$ have significant impact on the nonlinear vibrations of the entire structure. The increase in the mass of the element $M$ and the amplitude of the vibrations result in a decrease in the natural frequency of the beam. It has also been shown that systems with shorter lengths are much more susceptible to change the above parameters. Analysis of the beam-mass system presented in this work can be developed in the future, in particular of the impact analysis of the rigidity of the connection between the piston rod and the cylinder or latch system.

\section{Acknowledgments}

This study was carried out within the statutory funds of the Czestochowa University of Technology (BS/PB 1-100/3010/2019/P).

\section{References}

[1] A.L. Gawali, S.C. Kumawat, World Res. J. Civ. Eng. 1, 15 (2011).

[2] R.D. Firouz-Abadi, M. Rahmanian, M. Amabili, J. Vib. Acoust. 135, 051017 (2013).

[3] K. Sokół, J. Vibroeng. 20, 1299 (2018).

[4] S. Uzny, Ł. Kutrowski, T. Skrzypczak, Vib. Phys. Syst. 30, 2019211 (2019).

[5] L. Majkut, J. Theor. Appl. Mech. 47, 193 (2009).

[6] J. Hong, J. Dodson, S. Laflamme, A. Downey, Appl. Sci. 9, 2996 (2019).

[7] S. M. Hozhabrossadati, A. Aftabi Sani, M. Mofid, J. Vib. Control 22, 955 (2016).

[8] M. Chajdi, A. Adri, K. El Bikri, R. Benamar, Diagnostyka 20, 111 (2019).

[9] M. Infantes, P. Vidal, R. Castro-Triguero, L. Gallimard, E. Garcia-Macias, O. Poliot, Mech. Adv. Mater. Stuct. 26, 1 (2019).

[10] G.R. Gillich, P.Z Iosif, M. Abdel Wahab, N. Gillich, I.C. Mituletu, C. Nitescu, Shock Vib. 2016, 2086274 (2016).

[11] Q. Mao, Math. Comput. Modell. 54, 756 (2011). 